\title{
Structure and magnetic properties of multi-walled carbon nanotubes modified with iron
}

\author{
G.E. Grechnev ${ }^{1}$, V.A. Desnenko ${ }^{1}$, A.V. Fedorchenko ${ }^{1}$, A.S. Panfilov ${ }^{1}$, L.Yu. Matzui ${ }^{2}$, \\ Yu.I. Prylutskyy ${ }^{2,3}$, M.I. Grybova ${ }^{2}$, U. Ritter ${ }^{3}$, P. Scharff $^{3}$, and Yu.A. Kolesnichenko ${ }^{1}$ \\ ${ }^{1}$ B. Verkin Institute for Low Temperature Physics and Engineering of the National Academy of Sciences of Ukraine, \\ 47 Lenin Ave., Kharkov 61103, Ukraine \\ E-mail: grechnev@ilt.kharkov.ua \\ ${ }^{2}$ Kyiv National Taras Shevchenko University, Physical and Biological Faculties, \\ 64 Volodymyrska Str., Kyiv 01601, Ukraine \\ ${ }^{3}$ Technical University of Ilmenau, Institute of Physics, Laboratory of Chemistry, 09684 Ilmenau, Germany
}

Received July, 14, 2010

\begin{abstract}
Magnetic properties of multi-walled carbon nanotubes modified with iron (MWCNT+Fe) are studied in detail in the temperature range 4.2-300 K. Carbon encapsulated Fe nanoparticles were obtained using chemical vapor deposition method. The low-temperature SQUID magnetization measurements are supplemented by structural investigations with thermogravimetric (TG) analysis, transmission electron microscopy (TEM), $\mathrm{x}$-ray diffraction spectroscopy (XRD), and scanning electron microscopy (SEM). The magnetic susceptibility of MWCNT $+\mathrm{Fe}$ was also studied above room temperature to provide a complete picture of magnetic phase transitions.
\end{abstract}

PACS: $75.30 . \mathrm{Cr}$ Saturation moments and magnetic susceptibilities;

75.75.-c Magnetic properties of nanostructures;

81.05.U- Carbon/carbon-based materials;

81.07.De Nanotubes.

Keywords: carbon nanotubes, magnetic nanoclusters, magnetization, susceptibility.

\section{Introduction}

Carbon nanotubes (CNT) exhibit unique physical properties $[1,2]$, in particular, they are chemically and thermally stable, characterized by high mechanical strength, thermal and electrical conductivity, large specific surface area. Now CNT are regarded as the most attractive building blocks for nanoelectronics: they are able to form a perfect spin-transport medium, since electron transport in them is one-dimensional and ballistic with a long spin relaxation time and weak spin-orbital effects. Also, even pure CNT, which are nonmagnetic materials, are characterized by giant magneto-resistance $[3,4]$. On the other hand, it is quite obvious that modification of CNT (intercalation, chemical surface modification, filling the internal cavities with different elements) would lead to significant differences in their electronic structure and properties [5-7]. In particular, the encapsulated $\mathrm{Fe}$ nanowires are expected to exhibit excellent magnetic properties like the uniaxial magnetic anisotropy and the high magnetic coercitivity, which is larger than the coercitivity of bulk iron. Due to the very large magnetic shape anisotropies, the encapsula- tion of magnetic phases in CNT could provide a feasible approach to achievement of magnetic order stabilization against thermal fluctuations in systems having extremely reduced dimensions. Also, the ferromagnetic nanoclusters are expected to have much better magnetic properties than bulk metals due to the single domain nature [8].

Therefore, it is desirable to produce CNT with magnetic material inside tubes in a specific and controlled way. Beyond the geometrical advantage of a quasi-one-dimensional CNT design, the carbon shells can provide an effective protection against oxidation. It is especially important, since applications of ferromagnetic nanoclusters are limited due to air oxidation. Finally, except for the interest related to practical applications, a study of these magnetic systems provides an avenue for the exploration of the physics of the magnetic order in close-to-one-dimensional structures. In particular, a number of fundamental questions can be addressed about the role of spin degrees of freedom in the quantum wires or Luttinger liquid, where specific effects associated with the spin-charge separation are expected. 
Thus, the purpose of this work was to study the structure of multi-walled CNT (MWCNT) modified with iron and their magnetic characteristics (magnetic susceptibility and magnetization) as functions of magnetic field and temperature. By this way, it was expected to reveal and distinguish different iron phases and their spatial distribution within in-house fabricated MWCNT with encapsulated Fe.

\section{Experimental}

Chemical vapor deposition (CVD) was used to produce CNT filled with iron. In thermal CVD a conventional heat source is used, like a resistive or inductive heater, a furnace or an IR lamp. Basically, the growth process involves heating a catalyst material to high temperatures in a tube furnace. It was followed by flowing a hydrocarbon gas, being the carbon source, through the tube reactor for a chosen growth time. Then, under the flow of an inert gas to prevent oxidation of the carbon species, the system was cooled down to the room temperature. Finally, the carbonaceous materials grown over the catalyst have been collected.

In the course of CNT growth the key parameters are the chosen hydrocarbons, catalysts and the growth temperature. They are all together responsible for the properties of the obtained tubes. Our method is based on the catalytic decomposition of benzene (as the carbon source) and ferrocene (source of iron) in a tube furnace at different temperatures. To fill the reactor volume simultaneously with the components, the experimental set-up consists of an aerosol-generator and a tube furnace. The quartz glass tube (length: $70 \mathrm{~cm}$, diameter: $3 \mathrm{~cm}$ ) is preheated to a temperature between 800 and $950{ }^{\circ} \mathrm{C}$. The aerosol generator is made from quartz glass and the function is based on a spray-aerosol principle. Argon was used as a carrier gas for feeding the reactor with an aerosol of benzene and ferrocene. The aerosol flow rate is varied between 4 and $5 \mathrm{~L} / \mathrm{min}$ argon. In the process the ferrocene decomposes and provides the iron particles required for the nucleation of the CNT. After the reaction iron clusters are found inside of the CNT, whereas the aligned nanotubes grow on the quartz glass reactor wall. The reaction time is mostly responsible for the length of the tubes. The CNT, which are used in the experiments, were produced during the reaction time of 10-20 min. At the end of the reaction the aerosol generator is shut down and the reactor is cooled in an argon flow $(0.5 \mathrm{~L} / \mathrm{min}$; cooling time is $5 \mathrm{~h})$. Besides of the furnace temperature and the flow rate, the ferrocene concentration has the largest effect on diameters of the CNT, as well as the iron clusters found inside the nanotubes. The highest concentration of ferrocene we used was $5 \mathrm{wt} \%$, but the concentration can be reduced to a level of $0.2 \mathrm{wt} \%$. Such low concentrations of ferrocene lead to a decreased amount of iron inside the tubes, which is estimated from the thermogravimetric (TG) analysis and different transmission electron microscopy (TEM) pictures.
A high concentration of ferrocene results in the crystallization of ferrocene on the spray nozzle and reduces the possible reaction time to a few minutes. Also, the higher ferrocene concentration leads to greater number of iron clusters in the reactor, and the formed iron nanoclusters have a bigger diameter. The diameter of the formed CNT appeared to be directly related to the iron cluster diameter. Therefore one can obtain an increased CNT diameter by increasing the ferrocene concentration.

The structure and phase composition were studied by the thermogravimetric analysis (TG, Satorius), x-ray diffraction spectroscopy (XRD) with an x-ray diffractometer (Bruker AXS), scanning electron microscopy (SEM, Philips FEI), and transmission electron microscopy (TEM, Philips TECNAI).

In the low-temperature range $(4.2-290 \mathrm{~K})$ the magnetic properties of the iron filled CNT were studied by an inhouse SQUID magnetometer in the fields up to $50 \mathrm{kOe}$. For magnetization studies the sample was prepared by compacting the Fe-encapsulated CNT powder inside an elongated aluminum foil cylinder with diameter of $1.5 \mathrm{~mm}$ and about $7 \mathrm{~mm}$ in length. For all measurements the applied magnetic field was aligned along the cylinder axis in order to minimize the effect of demagnetization factor in magnetization data. The magnetization measurements were made after cooling the sample from room to helium temperatures both in zero magnetic field (zero-field-cooled, ZFC) and in the measured field (field-cooled, FC). Then the temperature dependences of the magnetization $M(T)$ were measured under a slow heating with the rate of about $1.5 \mathrm{~K} / \mathrm{min}$ in applied magnetic fields $H=0.3,5$ and $30 \mathrm{kOe}$. The field dependence of the magnetization was also measured at $T=4.2 \mathrm{~K}$ for $\mathrm{ZFC}$ regime in magnetic fields up to $40 \mathrm{kOe}$.

The magnetic susceptibility of MWCNT+Fe was also measured above room temperature to provide a complete picture of magnetic phase transitions. The Faraday technique upon heating and cooling of the specimens has been employed in magnetic field about $20 \mathrm{kOe}$. The heating and cooling rates did not exceed $10 \mathrm{~K} / \mathrm{min}$.

\section{Results and discussion}

The TG results in Fig. 1 show that the overall iron amount in CNT sample is about $4.6 \mathrm{wt} \%$. Also, the TG data demonstrate that quality of the obtained CNT is very high, and the amount of amorphous carbon is very low. The decomposition of the CNT starts at temperature about $570{ }^{\circ} \mathrm{C}$.

According to the electron microscopic results in Figs. 2 and 3, the obtained CNT are MWCNT. The inner diameter of MWCNT is $5-8 \mathrm{~nm}$, whereas the outer diameter is about $40-60 \mathrm{~nm}$ and the length is up to $100 \mu \mathrm{m}$. This MWCNT's contain clusters of iron (or its compounds) with average diameter $10-15 \mathrm{~nm}$ and length $120-140 \mathrm{~nm}$. 


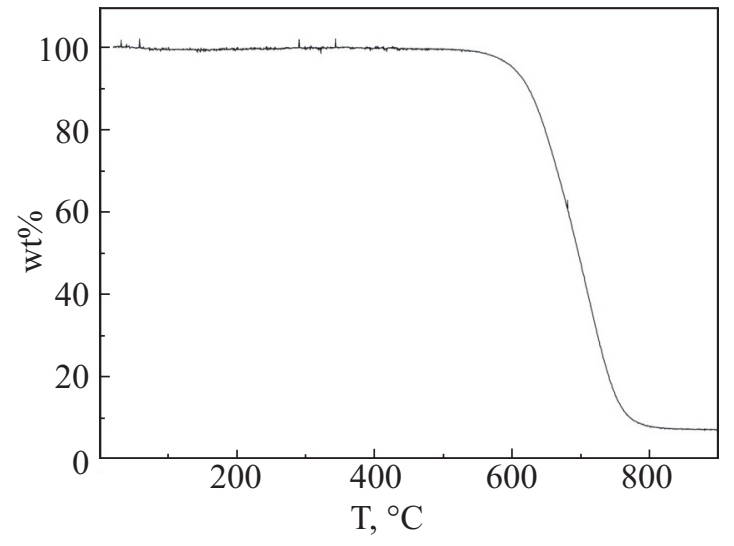

Fig. 1. Thermogravimetric measurements of MWCNT (starting mass $10.45 \mathrm{mg}$, rate $1 \%$ min in air).

The x-ray diffraction patterns of MWCNT grown with benzene (BZ) are displayed in Fig. 4. The peak at the angle of $26.1^{\circ}$ corresponds to the interplanar spacing between the CNT walls and is marked as CNT (002). The interlayer spacing of $3.411 \AA$ for nanotubes grown with BZ slightly exceeds that observed for the perfect graphite $(3.354 \AA)$. The intensity of the CNT (002) reflection is stronger in case of more tangled nanotubes grown with benzene than with other carbon sources. The other distinctive characteristic of CNT is the peak at $2 \theta=42.7^{\circ}$, which corresponds to the (100) reflection [9]. A peak at the angle of $2 \theta=35.4^{\circ}$

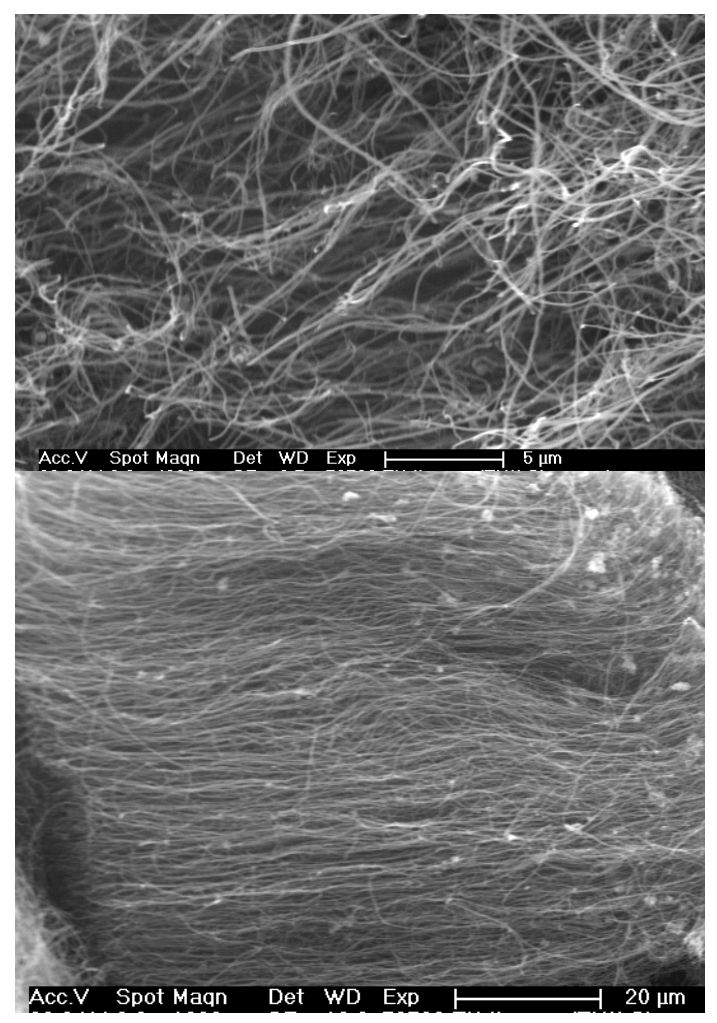

Fig. 2. SEM image of MWCNT modified with iron (ferrocene concentration is $1 \mathrm{wt} \%$ in benzene).

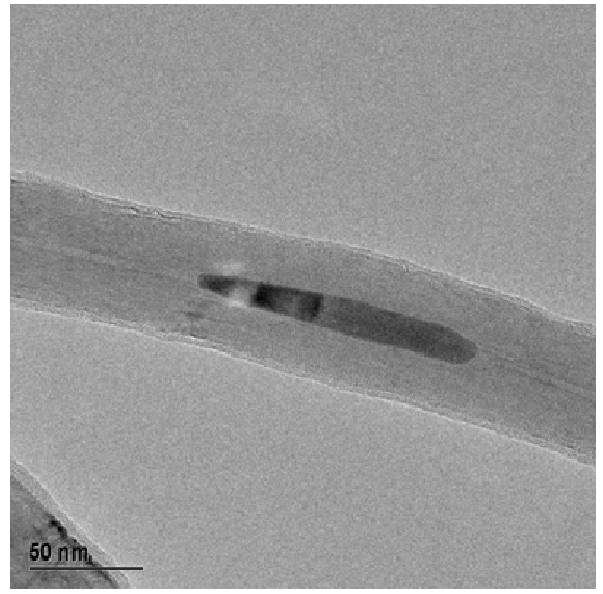

Fig. 3. TEM image of MWCNT modified with iron (ferrocene concentration is $1 \mathrm{wt} \%$ in benzene).

corresponds to the iron oxide (110) reflection [10]. The iron oxide (202) reflection at $42.6^{\circ}$ is superimposed by a much stronger CNT (100) feature. Another peak observed in the recorded patterns is the iron carbide peak, IC (103), at $2 \theta=$ $=44.8^{\circ}[10,11]$. This iron carbide phase is presumably a residue of CNT growth due to precipitation of carbon feed from catalyst/hydrocarbon drops [11]. Finally, the crystalline phase of $\alpha$-Fe is represented by a strong (110) reflexion, which is represented by additional peak at $51.1^{\circ}[10]$.

The temperature dependences of $\chi$ obtained at the low field of 300 Oe in both ZFC and FC regimes are presented in Fig. 5. The ZFC-W curve exhibits a conspicuous magnetic transition at temperatures slightly above $100 \mathrm{~K}$. The shape and position of this peculiarity resemble the socalled Verwey transition, which has been observed in the $\mathrm{Fe}_{3} \mathrm{O}_{4}$ oxide (magnetite) at $T_{m}=120 \mathrm{~K}$. This can be considered as indication of the presence of $\mathrm{Fe}_{3} \mathrm{O}_{4}$ nanoparticles in the studied sample. Moreover, the absence of distinct decay of magnetization with temperature, which is relevant for superparamagnetic systems, also points to a large magnetic anisotropy energy, as well as substantial fraction of magnetic nanoparticles. The discrepancy between ZFC and FC magnetization curves may imply a complex magnetic state at low temperatures, e.g., a spin-glass cluster state.

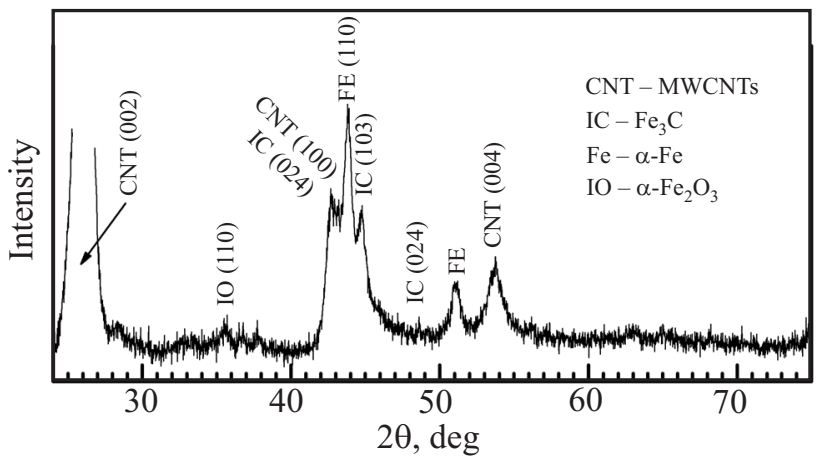

Fig. 4. XRD pattern for MWCNT modified with iron. 


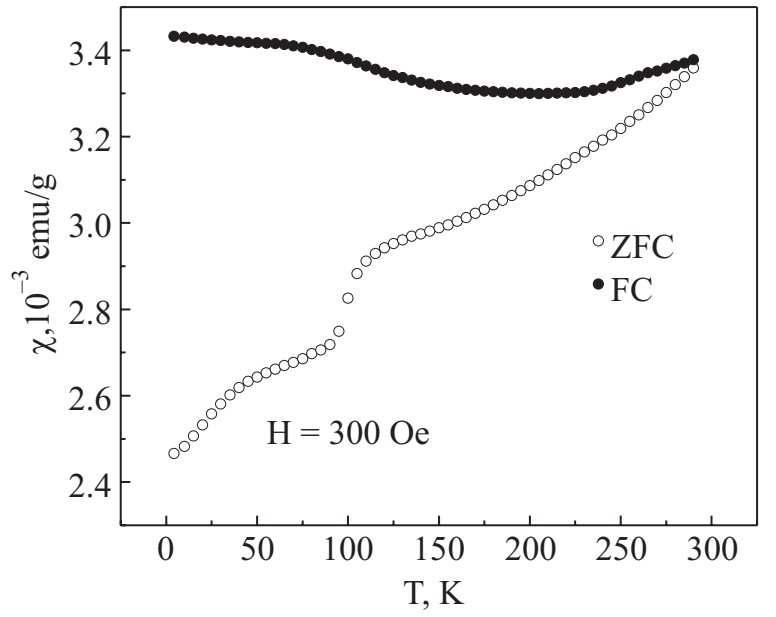

Fig. 5. Temperature dependence of magnetic susceptibility for MWCNT modified with iron measured in the range $4.2-300 \mathrm{~K}$ in the field 300 Oe for both FC and ZFC regimes.

The temperature dependence of magnetization at higher fields is given in Fig. 6. One can see a nonmonotonous step-like behavior of the temperature dependence of susceptibility $\chi(T)$ measured in the field $5 \mathrm{kOe}$ in the range 70-100 K. Such behavior can be attributed to appearance of some magnetic phase. It may be suggested, that this phase might appear in the iron nanoclusters, which resembles the recently suggested appearance of $\gamma$-iron phase [12]. On the other hand, the observed peculiarity may be related to a manifestation of exchange bias in larger particles. The measured temperature dependence of magnetization in the field of $20 \mathrm{kOe}$ is also listed in Fig. 6.

The field dependence of magnetization was measured at $T=4.2 \mathrm{~K}$ for ZFC regime and presented in Fig. 7. According to the high-fields magnetization data in Fig. 7, the value of saturated magnetic moment at the liquid helium temperature amounts to $8.5 \mathrm{emu} / \mathrm{g}$, to be compared with the

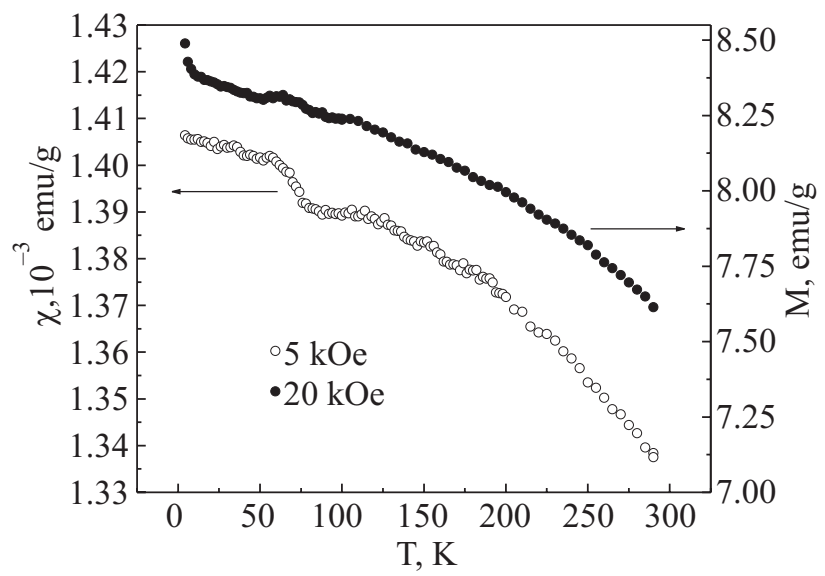

Fig. 6. Temperature dependences of magnetic susceptibility and magnetization for MWCNT modified with iron measured in the range $4.2-300 \mathrm{~K}$ for $\mathrm{ZFC}$ regime in the fields of 5 and $20 \mathrm{kOe}$, correspondingly.

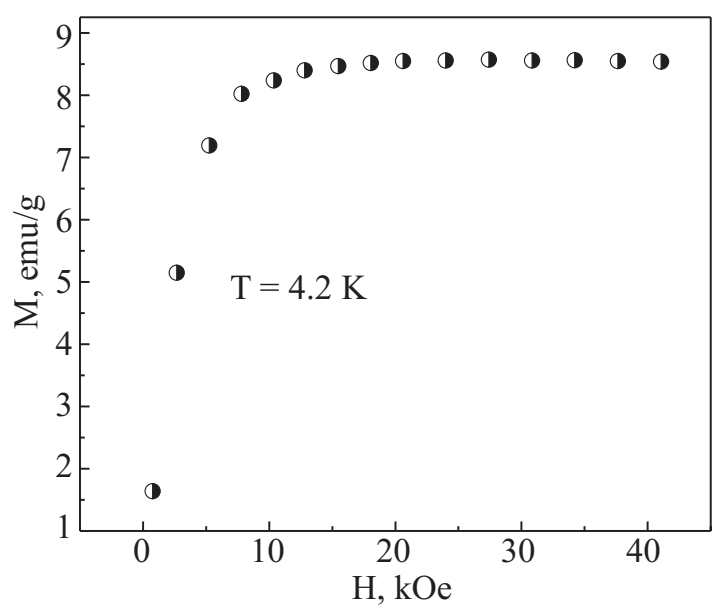

Fig. 7. Field dependence of magnetization for MWCNT modified with iron measured at temperature $4.2 \mathrm{~K}$ for $\mathrm{ZFC}$ regime.

value of $217 \mathrm{emu} / \mathrm{g}$ for saturation magnetization of bulk iron. If one assumes, that encapsulated nanoparticles consist predominantly of iron, one can estimate the share of iron containing phase as $4 \%$. The iron concentration in the sample was also estimated from the TG analysis as $(4 \pm 1) \mathrm{wt} \%$. This result agrees with our magnetization analysis, which presumably yields the content of magnetic material with a higher precision. Since the saturation appears at fields just above $10 \mathrm{kOe}$, as is seen in Fig. 7, this indicates a large magnetic anisotropy of the studied Fe-CNT system.

The temperature dependences of coercitivity $H_{C}$ and residual magnetization $M_{R}$ of our Fe-CNT sample were derived from the reversible curves of saturation magnetization and presented in Figs. 8 and 9. It is clearly seen the increase of $H_{C}$ and $M_{R}$ values with decreasing temperature. In particular, when temperature falls from 300 to $4.2 \mathrm{~K}$, the coercitivity has increased by 2.3 times, and the residual magnetization also increased by 1.5 times. At helium temperatures the values of $H_{C}$ and $M_{R}$ are equal to $1120 \mathrm{Oe}$ and $2.95 \mathrm{emu} / \mathrm{g}$, respectively.

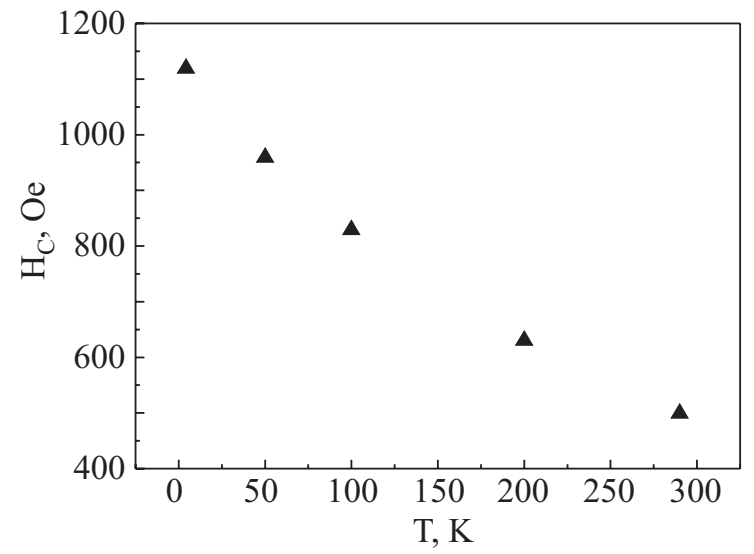

Fig. 8. Temperature dependence of coercitivity for MWCNT modified with iron in the range $4.2-300 \mathrm{~K}$. 


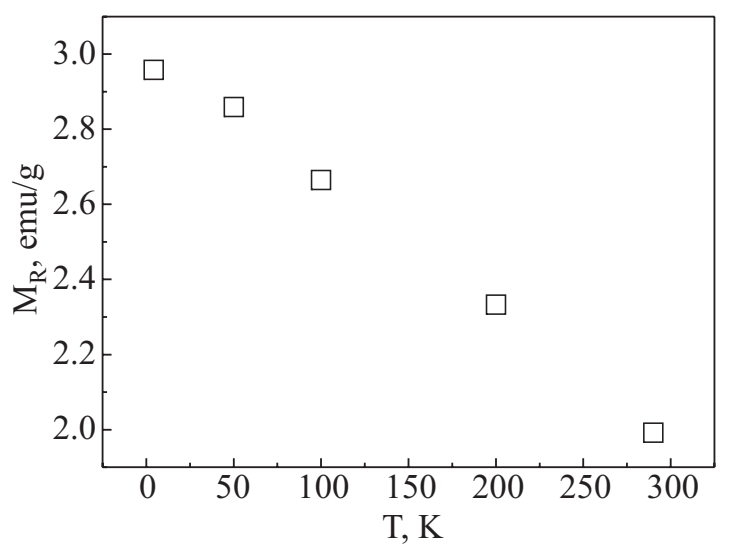

Fig. 9. Temperature dependence of residual magnetization $M_{R}$ for MWCNT modified with iron in the range 4.2-300 K.

The results of a supplementary study of magnetic susceptibility $\chi(T)$ dependencies above room temperature are given in Fig. 10. This figure clearly shows a magnetic transition at about $480 \mathrm{~K}$, which is presumably related to the phase of cementite, with the Curie temperature of about $483 \mathrm{~K}$ for the bulk $\mathrm{Fe}_{3} \mathrm{C}$ [13]. At higher temperatures one can expect superparamagnetic contribution from iron nanoclusters with the blocking temperature of about $500 \mathrm{~K}$.

\section{Conclusions}

The Fe-MWCNT nanoclusters as produced by the method of the catalytic decomposition of benzene and ferrocene were investigated with respect to their structural and magnetic characteristics. The SEM and TEM studies show that MWCNT has the inner diameter about 5-8 nm, whereas the outer diameter is about $40-60 \mathrm{~nm}$ and the length is up to $100 \mu \mathrm{m}$ and contains clusters of iron (or its compounds) with average diameter 10-15 nm and length 120 $140 \mathrm{~nm}$. The x-ray diffraction study has revealed a number of iron and carbon phases present in Fe-MWCNT. The results of investigation of $\mathrm{ZFC}$ and $\mathrm{FC}$ temperature depen-

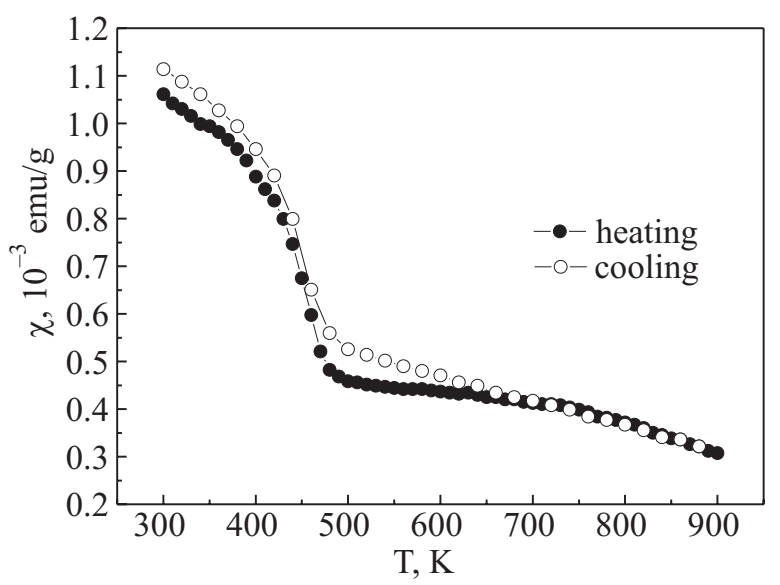

Fig. 10. Temperature dependence of magnetic susceptibility for MWCNT modified with iron above room temperature. dences of magnetic susceptibility indicate a number of magnetic phase transitions in the studied Fe-MWCNT system, including the Verwey transition in $\mathrm{Fe}_{3} \mathrm{O}_{4}$ particles, presumably attached outside MWCNT. It was shown that both residual magnetization $M_{R}$ and coercive field $H_{C}$ decrease monotonically with increasing temperature. The existence of a spin-glass state and interactions between nanoparticles of various sizes could contribute to the observed magnetic behavior.

1. M.S. Dresselhaus, G. Dresselhaus, and P.C. Eklund, Science of Fullerenes and Carbon Nanotubes, New York: Academic Press (1996).

2. P.J.F. Harris, Carbon Nanotubes and Related Structures, Cambridge: Univ. Press (1999).

3. G.C. McIntosh, G.T. Kim, J.G. Park, V. Krstic, M. Burghard, S.H. Jhang, S.W. Lee, S. Roth, and Y.W. Park, Thin Solid Films 417, 67 (2002).

4. A. Cottet, T. Kontos, S. Sahoo, H.T. Man, M.S. Choi, W. Belzig, C. Bruder, A.F. Morpurgo, and C. Schonenberger, Semicond. Sci. Techn. 21, 78 (2006).

5. P.K. Tyagi, A.M. Manoj, K.S.D. Misraa, J. Ghatak, P.V. Satyam, and F. Le Normand, Appl. Phys. Lett. 86, 253110 (2005).

6. E. Borowiak-Palen, M.H. Ruemmeli, T. Gemming, T. Pichler, R.J. Kalenczuk, and S.R.P. Silva, Nanotechn. 17, 2415 (2005).

7. O.V. Mykhailenko, D. Hui, Y.M. Strzhemechny, D. Matsui, Yu.I. Prylutskyy, and P. Eklund, J. Comput. Theoret. Nanosci. 4, 1 (2007).

8. N. Grobert, W.K. Hsu, Y.Q. Zhu, J.P. Hare, H.W. Kroto, D.R.M. Walton, M. Terrones, H. Terrones, P. Redlich, M. Ruehle, R. Escudero, and F. Morales, Appl. Phys. Lett. 75, 3363 (1999).

9. A.G. Kudashov, A.V. Okotrub, L.G. Bulusheva, I.P. Asanov, Y.V. Shubin, and N.F. Yudanov, J. Phys. Chem. B108, 9048 (2004).

10. S.A. Ibitoye and A.A. Afonja, J. Miner. Mater. Characterization and Engineering 7, 203 (2008).

11. U. Narkiewicz, N. Guskos, W. Arabczyk, J. Typek, T. Bodziony, and W. Konicki, Carbon 42, 1127 (2004).

12. A. Khasanov, J. He, J. Gaillard, K. Yang, A.M. Rao, C.M. Cameron, J.M. Schmeltzer, J.G. Stevens, and A. Nath, Appl. Phys. Lett. 93, 013103 (2008).

13. I.V. Ovsienko, L.Yu. Matzui, N.I. Zakharenko, N.G. Babich, T.A. Len, Yu.I. Prylutskyy, D. Hui, Yu.M. Strzhemechny, and P.C. Eklund, Nanoscale Res. Lett. 3, 60 (2008). 